\title{
A Rare Case of Basidiobolomycosis that has an Excellent Response with Itraconazole: Case Report
}

\author{
Riezky Januar Pramitha ${ }^{\mathrm{a}}$, Yuri Widia ${ }^{\mathrm{b}}$, Linda Astari ${ }^{\mathrm{b}}$, Sawitri $^{\mathrm{b}}$, Evy Ervianti ${ }^{\mathrm{b}}$ \\ riezky.januar.pramitha@gmail.com
}

${ }^{a}$ Resident, Dermatology and Venereology Department Faculty of Medicine Universitas Airlangga, Dr. Soetomo Teaching Hospital Jl. Prof. dr. Moestopo 6-8, Surabaya 60286, Indonesia

${ }^{b}$ Dermatologist, Dermatology and Venereology Department Faculty of Medicine Universitas Airlangga, Dr. Soetomo Teaching Hospital Jl. Prof. dr. Moestopo 6-8, Surabaya 60286, Indonesia

\begin{abstract}
Background: Basidiobolomycosis due to Basidiobolus ranarum is an uncommon subcutaneous tissue fungal infection in immunocompetent patients. General practitioners frequently make mistake in diagnosing this skin condition because of its relentless growth, painless, slow, and no response against antibiotics and anti-inflammatory drugs, and they are not familiar with these lesions.

Case: A 23 years-old man was consulted from the pulmonary department with progressive painless, non-tender multiple nodules and swelling over his back, chest, and arm in the last 1,5 year ago which was initially misdiagnosed as cutaneous tuberculosis. Extensive investigation performed with excision biopsy and culture examination revealed the presence of Basidiobolus ranarum. After initiating antifungal therapy with itraconazole, the patient's clinical condition improved significantly.

Conclusion: Basidiobolomycosis is a disease that is likely to be cured, but misdiagnosis possibility is high. Early diagnosis and knowledge of this condition are very important. There was a dramatic improvement after 4 weeks of oral itraconazole $400 \mathrm{mg}$ daily and complete resolution was reached after 12 weeks of treatment.
\end{abstract}

Keywords: Basidiobolomycosis; Basidiobolus ranarum; subcutaneous nodule; itraconazole; misdiagnose

\section{Introduction}

Basidiobolomycosis due to Basidiobolus ranarum is an uncommon subcutaneous tissue fungal infection. It might cause variable clinical findings such as subcutaneous and gastrointestinal findings, and sometimes acute systemic illness (El-Shabrawi et al., 2014); (Shaikh et al., 2016); (Raquel Vilela and Mendoza, 2018). The first case of Basidiobolomycosis in Indonesia had been reported in 1956 (Lie Kian Joe et al., 1956).

Basidiobolus ranarum fungus can be found in soil, decayed vegetables, andreptiles, amphibians, insectivorous bats, and fish intestines (Shaikh et al., 2016); (Mule et al., 2018). Basidiobolomycosis can occur worldwide, reported mostly from tropical and subtropical areas. Children and young adults are commonly affected (80\% below 20 years age). More males are affected than females (Kumaravel et al., 2016); (Thomas J. Walsh, Hayden and Larone, 2018). 
Basidiobolomycosis is often misdiagnosed as another mycosis (sporotrichosis and pythiosis), infection by parasites (onchocerciasis and filarial elephantiasis), infection by bacteria (Mycobacterium ulcerans, Mycobacterium tuberculosis, and chronic abscesses), and another disease, including soft tissue tumor and Burkitt's lymphoma (Barati et al., 2010). There are several pharmalogical agents that are known to have good efficacy against Basidiobolus infection, which are: trimethoprim-sulfamethoxazole, amphotericin B, potassium iodide (KI), oral azoles, and potassium iodide in combination with oral azoles (Geramizadeh et al., 2012); (Shaikh et al., 2016). We reported a patient with Basidiobolomycosis that had excellent response to itraconazole-only treatment.

\section{Case Presentation}

A 23 years-old man who worked as a carpenter in Kalimantan Forest, Indonesia for 5 years was consulted from the pulmonary department with progressive multiple painless bumps and swelling that appeared in his back in the last 2 years ago. The bump increase in size and spread to the chest, arms, and neck (Fig.1).

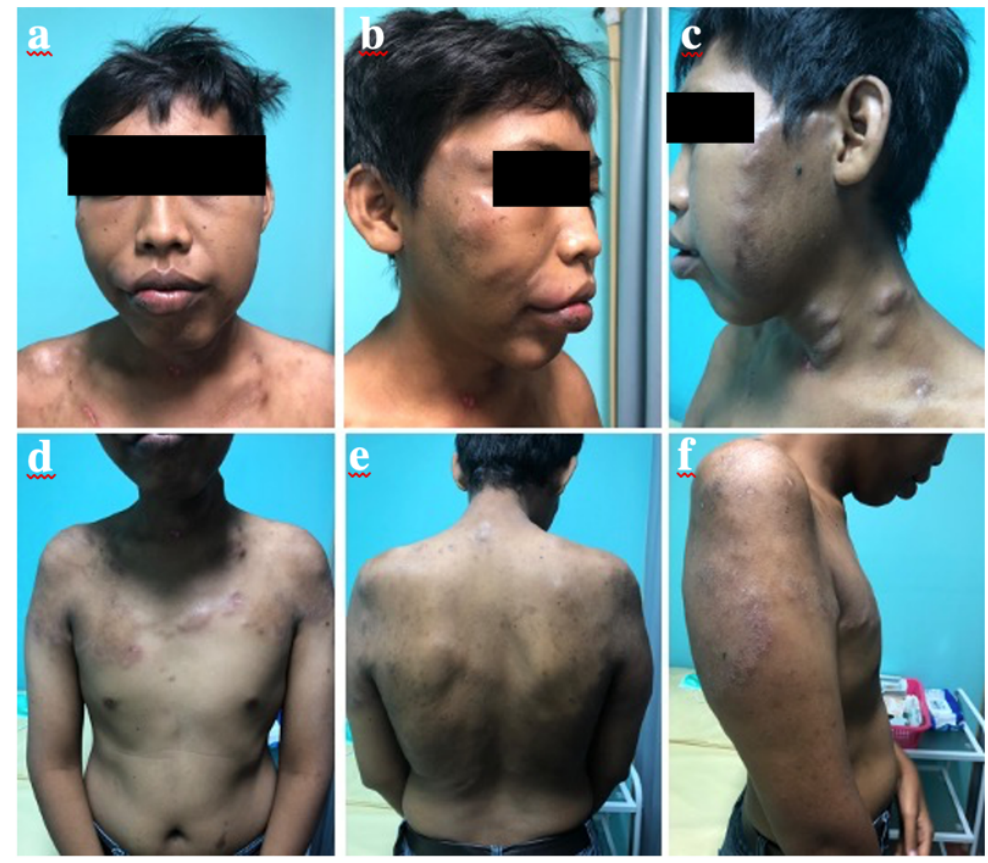

Fig 1. Clinical presentation of the patient (a), (b), and (c) Facial primarily affecting the midline of the face primarily involving both cheek, neck, upper and lower lip; (d) and (e) front and back chest; (f) right and left upper arm revealed multiple nodules, tenderness, mobile, and firm consistency 
Extensive investigation of his clinical condition did not result in a conclusive diagnosis. Several Fine Needle Aspiration Biopsy (FNAB) was performed on another hospital that exhibited only granulomatous inflammation and he was initially misdiagnosed as cutaneous tuberculosis. The patient got an antituberculosis drug oral, but his clinical situation deteriorated progressively.

On general examination, there was no regional lymphadenopathy. From dermatological state revealed, generalized edema of the face that was more pronounced in the midline. On the lateral aspect of the face, neck, left and right upper arm and front and back chest showed multiple nodules vary in size between $1.5 \times 2$ $\mathrm{cm}$ to $2.5 \times 3 \mathrm{~cm}$. Nodule and the swelling were painless, firm in consistency but some part began to indurate with rounded and smooth edges and had free mobility over the underlying structures. The overlying skin was hyperpigmented with fine scales. There was reactive in HBsAg test and HIV status was negative.

Management in this patient was performed in multidisciplinary care. The pulmonary department was examined the sputum specimen and tuberculin test and the result was negative for Mycobacterium tuberculosis. The Gastro-Entero-Hepatology Division in Internal Department performed FibroScan examination and abdominal ultrasonography and there is no abnormality. The Ear, Nose, and Throat (ENT) department examined waters and skull AP/lateral of the nasal sinuses and was assessed with mild maxillary bilateral sinusitis. The dermatology department was performed an excision biopsy of the skin nodule using Periodic acid Schiff (PAS) stain. The result demonstrated aseptate hyphae that was surrounded by an uncommon eosinophilic mass (Splendore - Hoeppli phenomenon) (Fig. 2). Microbiological investigations with culture on Sabouraud agar medium revealed that growth of these colonies was rapid (3-4 days) at $30^{\circ} \mathrm{C}$ showed flat and furrowed. The color of the macroscopic appearance was yellowish with pale reverse and waxy texture (7 days).
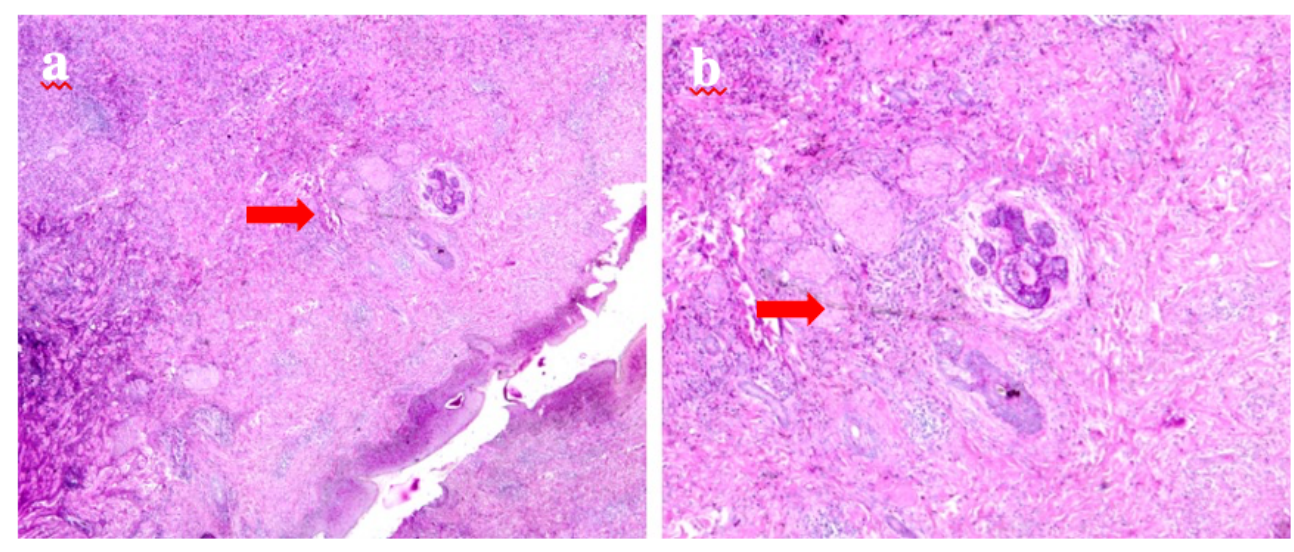

Fig. 2. Histopathology result (a) and (b) using PAS with 100x and 400x magnification showing aseptate hyphae 
Lactophenol cotton blue (LCB) wet mount demonstrated large vegetative hyphae (diameter of 8-20 $\mu \mathrm{m}$ ) forming numerous smooth, round (diameter of 20-50 $\mu \mathrm{m}$ ), and thick-walled zygospores (sexual spores) that had two closely beak-like appendages, which confirmed Basidiobolus ranarum as the type of fungus (Fig.3)
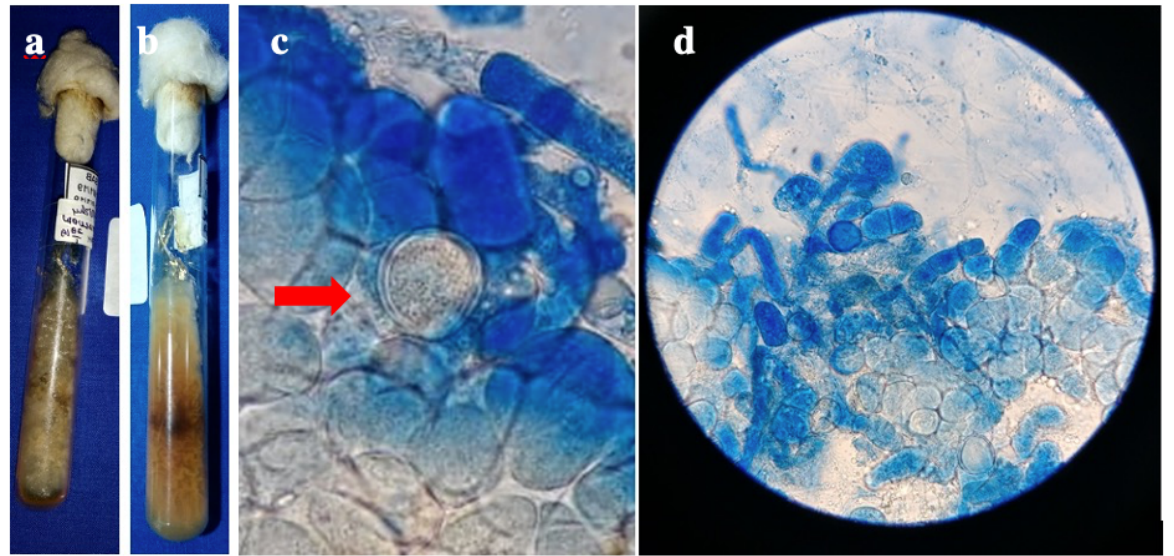

Fig. 3. (a) and (b) Culture specimens day 7 showed brown and radially folded; (b). Lacto Phenol Cotton Blue with 40x magnification showing zygospore; (c) Sporophore and Conidia of Basidiobolus ranarum

After the diagnosis was established, this patient was treated with itraconazole $400 \mathrm{mg}$ daily with divided doses. We are followed up with this patient every week. Itraconazole therapy was received well by the patient and lesion resolution began after the first month of therapy. There was a dramatic improvement with a decrease in facial puffiness and decreased in size of bump in his face, neck, chest, arm, and back after 4 weeks of oral itraconazole $400 \mathrm{mg}$ daily and complete resolution was reached after 12 weeks of treatment (Fig.4).
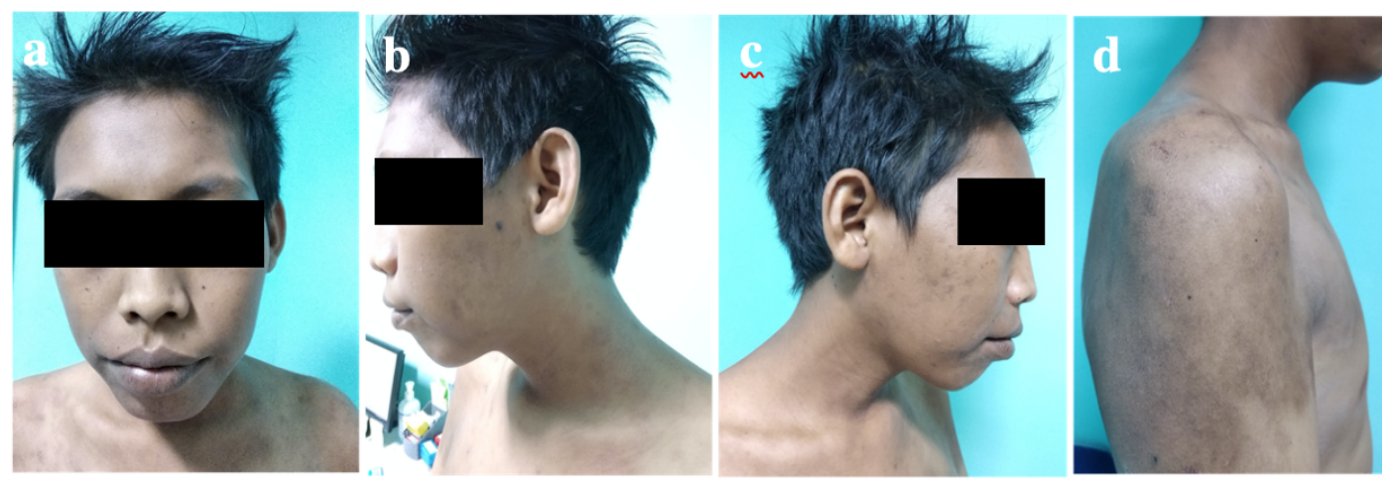

Fig. 4. (a-d) Facial puffiness dramatic improvement, the nodule was absent and there is only hyperpigmentation lesion on his face, neck, and limbs after 12 weeks treatment. 
To evaluate the success of the treatment we performed a re-excision biopsy for confirmation by histopathology and microbiological fungal culture and as the result, there was no fungal agent found anymore. We continued the treatment until one month following the negative fungal result.

\section{Discussion}

Basidiobolous ranarum is a filamentous fungus belonged to Zygomycetes class, Entomopthorales order, Basidiobolaceae family and Basidiobolus genus (El-Shabrawi et al., 2014); (Shaikh et al., 2016). Its port-ofentry is thought to be injured skin caused by scratches, insect bites, and minor cuts (Al Jarie et al., 2011); Barati et al., 2010). The patient's history of work as a carpenter in the Kalimantan forest could be identified as a predisposing factor in our case. Subcutaneous basidiobolomycosis is the commonest presentation that typically presents as a slowly progressive subcutaneous nodule developing over time into a large, firm, rubbery, indurated, painless, and freely mobile plaque-like in our case. Clinically these infections present as subcutaneous nodules affecting the gluteal, but some studies showed that even it is rare, the lesion can also find on the face, neck, and shoulder like in this case (Geramizadeh et al., 2012); (Shaikh et al., 2016).

Definitive diagnosis was confirmed by microscopy and culture of deep lesional biopsy. Histopathological examination commonly revealed an inflammatory infiltrate which is mixed with histiocytes, eosinophils, lymphocytes, neutrophils, and Splendore-Hoeppli phenomenon (dense eosinophilic granular infiltrates surrounding hyphae element). Subcutaneous tissue biopsy revealed broad, thin-walled hyphae found with concomitant acute and/or chronic inflammatory cell infiltrates. These aseptate hyphae usually appears without cytoplasm (Barati et al., 2010); (Shaikh et al., 2016).

Rate of growth Basidiobolus ranarum is fast, has maturity period of 5 days, and might grow more rapidly at $30^{\circ} \mathrm{C}$. Basidiobolus might be cultivated on standard mycology media such as potato cornmeal, dextrose, Sabouraud agar. Their colonies are furrowed and flat, have yellowish to greyish surface with a pale reverse, and have waxy texture. Sometimes, satellite colonies are formed through ejected sporangial germination which showed radial folds and using Lactophenol cotton blue wet mount demonstrated several zygospores that are commonly thick-walled, smooth, and had distinct conjugation beaks, which are the characteristics of Basidiobolus ranarum. In this patient, we performed microbiological investigations with culture on Sabouraud agar medium that growth of these colonies on 3-4 day and the color of macroscopic appearance with pale reverse and waxy texture on 7 days (Shaikh et al., 2016); (Raquel Vilela and Mendoza, 2018).

Pharmacological agents that are known to have good efficacy against Basidiobolus infection include trimethoprim-sulfamethoxazole, amphotericin B, potassium iodide (KI), oral azoles, and potassium iodide in 
combination with oral azoles. Itraconazole or KI might be feasible as first-line drug-of-choices, but KI was not available in our setting, and because there were multiple lesions, treatment with triazole especially itraconazole seems to be most appropriate choice in terms of treatment response and safety. Itraconazole is a first-generation antifungal which is highly hydrophobic and has high molecular weight. Itraconazole has an antifungal activity as fungicidal with a broader range. Cytochrome P450(CYP)-dependent 14- $\alpha$-demethylase (CYP51 or Erg 11p) enzyme, which catalyzes targeted synthetic reaction, is this drug's target. Oral itraconazole has also been reported to produce good results at a dose of (200-400 mg/day). Higher dose of than common therapeutic dose therapy is required to reach treatment results and prolonged daily therapy, where researchers suggested for months, because the organism exhibits relative resistance to antifungals (Mule et al., 2018); (Raquel Vilela and Mendoza, 2018). In our case, oral itraconazole $400 \mathrm{mg}$ daily gave excellent response and complete resolution after 12 weeks of treatment.

\section{Conclusion}

Basidiobolomycosis is a potentially curable disease. Diagnosis might be established through high suspicion index and tissue biopsy in appropriate time both for microbiological fungal cultures and histopathology might help early diagnosis and adequate therapy implementation. Regardless if an area is nonendemic of this condition, early diagnosis and knowledge are very important to avoid misdiagnosis, disfigurement, and unnecessary management. There was a dramatic improvement after oral itraconazole therapy.

\section{Acknowledgements}

I would like to dedicate my gratitude to all the lecturers of Dermatology and Venereology, Faculty of Medicine Airlangga University, my family, and my friends for their endless support and prayers

\section{References}

Al Jarie, A., Al Azraki, T., Al Mohsen, I., Al Jumaah, S., Almutawa, A., Fahim, Y. M., \& Hussein, M. R. A. E. (2011). Basidiobolomycosis: case series. Journal de mycologie médicale, 21(1), 37-45. https://doi.org/10.1016/j.mycmed.2010.11.002

Barati, M., TALEBI, T. M., Nojomi, M., \& Kerami, F. (2010). Ten-year experience of rhinocerebral zygomycosis in a teaching hospital in Tehran. Iranian Journal of Clinical Infectious Diseases, 5(3), pp. 117-120.

El-Shabrawi, M. H., Arnaout, H., Madkour, L., \& Kamal, N. M. (2014). Entomophthoromycosis: a challenging emerging disease. Mycoses, 57, 132-137. doi: 10.1111/myc.12248.

Geramizadeh, B., Kazemi, K., Shamsaifar, A. R., Bahraini, A., Nikeghbalian, S., \& Malekhosseini, S. A. (2012). Isolated renal mucormycosis after liver transplantation: an unusual case report. Iranian Red Crescent Medical Journal, $14(7), 447$. 
Kumaravel, S., Bharath, K., Rajesh, N. G., Singh, R., \& Kar, R. (2016). Delay and misdiagnosis of basidiobolomycosis in tropical South India: case series and review of the literature. Paediatrics and international child health,36(1), 52-57. doi: $10.1179 / 2046905514 Y .0000000140$.

Lie-Kian-Joe, N. I. T. E., Pohan, A., Van der Meulen, H., \& Emmons, C. W. (1956). Basidiobolus ranarum as a cause of subcutaneous mycosis in Indonesia. Arch. Derm., 74, 378-383.

Mule, P., Kelkar, R., Biswas, S., Bhat, V., (2018) ‘Subcutaneous Zygomycosis Masquerading As A Soft Tissue’, 7(4), pp. 6-9. doi: DOI: http://dx.doi.org/10.24327/ijcar.2018//dx.doi.org/10.24327/ijcar.2018.11489.1990.

Raquel Vilela and Mendoza, L. (2018) ‘Human pathogenic Entomophthorales', American Society For Microbilogy, 31(4), pp. 1-40. doi: https://doi.org/10.1128/CMR .00014-18.

Shaikh, N., Hussain, K. A., Petraitiene, R., Schuetz, A. N., \& Walsh, T. J. (2016). Entomophthoramycosis: a neglected tropical mycosis. Clinical Microbiology and Infection, 22(8), 688-694. doi: 10.1016/j.cmi.2016.04.005.

Thomas J. Walsh, Hayden, R. T. and Larone, D. H. (2018) Larone's Medically Important Fungi : a guide to identification. 6th edn. Washington, DC. doi: 10.1128/9781555819880. 